\title{
Misuse of amphetamines and related drugs
}

\section{Nicholas Seivewright \& Charles McMahon}

This article discusses the misuse of drugs which act as stimulants, an effect mainly produced by enhancement of the central transmission of catecholamines, particularly dopamine. Stimulant misuse is extremely widespread in the UK and elsewhere, but primary users of such drugs present relatively rarely for treatment at drug services. At present such services mainly see heroin users, not only because the greater addictiveness of heroin produces generally higher levels of problems, but because a substitute treatment can be used in that group in the form of methadone.

There are several reasons, however, why it is important to have a good working knowledge of stimulant misuse. Drug services consider amphetamine misuse a hidden epidemic, in which the heaviest users experience many of the same problems as heroin users without receiving similar treatment, and various policy initiatives are underway to attract this group into services.

Meanwhile in recent years cocaine usage has transferred to the much more potent form known as 'crack', which is causing severe problems in some of the UK's inner cities. Use of methylenedioxymethamphetamine (MDMA, 'ecstasy') by young people, associated with the 'rave' nightclub scene, is on a huge scale and the drug is proving to have some disturbing adverse effects in a minority of cases, including the well-publicised fatalities. Most importantly for psychiatrists, stimulant misuse is the form of drug misuse most associated with psychiatric complications, which often require management within general mental health services.

Table 1 describes the main stimulant drugs and indicates the ways in which they are used, approximate current street prices, and their classification under the 1971 Misuse of Drugs Act which dictates severity of penalties for the offences of possession or supplying.

\section{Amphetamine}

The form of amphetamine predominantly used by drug misusers is a powder, substantially adulterated with other substances, which is known as 'speed' or 'whizz'. The amphetamine contained is a racemic mixture of the $\mathrm{d}$ - and l-isomers, the lform being relatively inactive. Many recreational users simply swallow the drug, licking it from a finger or else putting it in a drink or swallowing an amount in cigarette paper, but it can also be snorted or injected. Sometimes pharmaceutical preparations are misused, the only preparation of amphetamine itself now routinely available being dexamphetamine sulphate tablets.

\section{Cocaine}

Cocaine hydrochloride is a white powder for which many claims have been made over the years proclaiming this form as a drug entirely compatible with an executive lifestyle. Such use is by snorting, while polydrug users also inject the powder, often along with heroin in the apparently inappropriately named 'speedball'. Starting in the US however, and now also in the UK, cocaine use is increasingly in the form of 'crack', a chemically altered preparation which is purer, more potent in its effects and withdrawal effects, and more likely to lead to heavy compulsive use. The chemical process is a simple one, involving heating with sodium bicarbonate. The appeal of the volatile crack is that

Dr Seivewright is Consultant Psychiatrist in Substance Misuse for the Specialist Mental Health Directorate, Norfolk House, 4 Norfolk Street, Sheffield. His main clinical and research interests include personality disorder, cocaine misuse, and pharmacological treatments in addictions. Dr McMahon is a Senior Registrar in Substance Misuse in Community Health Sheffield NHS Trust. He is particularly interested in the relationship between drugs of misuse and psychiatric disorder. 
Table 1. The main stimulant drugs of misuse

\begin{tabular}{|c|c|c|c|c|}
\hline Drug & Description & $\begin{array}{l}\text { Routes of } \\
\text { administration }\end{array}$ & $\begin{array}{l}\text { Street } \\
\text { price }\end{array}$ & $\begin{array}{l}\text { Misuse of Drugs } \\
\text { Act classification }\end{array}$ \\
\hline Amphetamine & $\begin{array}{l}\text { Usually light coloured powder, very low } \\
\text { purity (approx } 5 \% \text { ). Some pharmaceutical } \\
\text { preparations, e.g. dexamphetamine } \\
\text { sulphate tablets }\end{array}$ & $\begin{array}{l}\text { Swallowed, } \\
\text { snorted, IV }\end{array}$ & $\begin{array}{l}£ 10-15 \\
\text { per gram }\end{array}$ & $\begin{array}{l}B \text {, but } A \text { if prepared } \\
\text { for injection }\end{array}$ \\
\hline Cocaine $\mathrm{HCl}$ & $\begin{array}{l}\text { White powder, moderate purity } \\
\text { (up to } 50 \% \text { ) }\end{array}$ & Snorted, IV & $\begin{array}{l}£ 60-100 \\
\text { per gram }\end{array}$ & A \\
\hline Crack cocaine & Crystalline 'rocks' & Smoked, IV & $\begin{array}{l}£ 25 \text { per } \\
150 \text { mg rock }\end{array}$ & A \\
\hline MDMA & $\begin{array}{l}\text { Various manufactured tablets, often } \\
\text { with characteristic motifs }\end{array}$ & Swallowed & $\begin{array}{l}£ 15-25 \text { per } \\
100-120 \mathrm{mg} \\
\text { tablet }\end{array}$ & A \\
\hline
\end{tabular}

an intense euphoria is experienced rapidly by smoking, reflecting blood concentrations rising at a rate normally only seen after injecting drugs. Some individuals do inject crack, but this confers no major advantage. In some areas crack is becoming the only available form of cocaine, which polydrug users may inject if that is their preferred route of drug usage. Interestingly, the equivalent purified form of amphetamine, known as 'ice', is by contrast hardly encountered in the UK.

\section{Methylenedioxymethamphetamine (MDMA, 'ecstasy')}

This methylated amphetamine has a long history, but is currently popular as a recreational drug which has hallucinogenic properties as well as the stimulant effect. It appears in the form of tablets, which it is believed are mainly imported from other parts of Europe. Many have a characteristic motif or imprint, and users may try to seek out a type with which they are familiar, especially if they consider it produces genuine MDMA effects, as there are many bogus preparations in circulation. Some such imitations contain amphetamine and

Box 1. Effects and withdrawal effects of stimulant drugs

Effects

Early - increased energy, elation, reduced appetite

Late - overactivity, confusion, paranoia

Withdrawal effects Depression, irritability, agitation, craving, hyperphagia, sleep disturbances (hypersomnia, nightmares)
LSD, while a range of other drugs which produce hallucinations, such as the general anaesthetic agent ketamine, have been passed off as MDMA. Substances which on analysis prove to be chemically closely related analogues of MDMA also appear, produced in attempts to bypass specific drug legislation. Although MDMA users may also use other drugs, including amphetamine, MDMA is not reported to be used by methods other than swallowing the tablets.

\section{Others}

Other stimulant drugs which may be misused to a minor degree include the appetite suppressants, such as diethylpropion (Apisate, Tenuate Dospan) and fenfluramine (Ponderax), decongestants containing ephedrine or pseudoephedrine, methylphenidate (Ritalin), and the monoamine-oxidase inhibitor tranylcypromine (Parnate). While these may certainly be associated with clinical problems in individuals, there is little systematic evidence and they will not be discussed further here.

Of the illicit drugs, amphetamine and cocaine are similar enough in many aspects to be considered together. MDMA is significantly different in terms of epidemiology, type of usage, and clinical features and so is discussed separately.

\section{Amphetamine and cocaine}

\section{Epidemiology}

The true prevalence of any form of drug misuse is unknown, as it is a concealed illicit activity. Apart from enforcement statistics, two sources of data 
which are of interest are surveys and Drug Misuse Database (DMD) figures, the former because they probably come closest to true estimates of usage, and the latter because they are from treatment services.

Surveys necessarily collect limited information, commonly whether individuals have "ever used" various drugs. In UK surveys, up to $10-12 \%$ of young people reported having tried amphetamines by the age of 19 , while less than $1 \%$ had tried cocaine, a similar figure to heroin (Institute for the Study of Drug Dependence, 1994). It is of course important to know what proportion of such experimenters progress to regular use, but this is a relatively uncharted area, until users come into contact with treatment services.

The DMD system incorporates, but extends far beyond, the notification of addicts to the Home Office Addicts Index. Of the drugs discussed here, only cocaine is notifiable (along with opiates), but the DMDs record the use of all drugs in the previous month by anyone considered to be a problem drug misuser presenting to a range of medical and non-medical services, including community drug teams, street agencies, psychiatric drug misuse services, and general practitioners. This data is best referred to as 'treated prevalence'.

In the six months to September 1994, the last period for which full figures are available, out of a total of 19381 individuals presenting in England, $10 \%$ were using amphetamine as their main drug and $4 \%$ cocaine. An additional $9 \%$ and $11 \%$ respectively were using amphetamines and cocaine as secondary drugs, usually to opiates, confirming the common clinical impression of polydrug use. Of individuals using amphetamine, $46 \%$ were injecting it, with considerable regional variation. Undoubtedly this figure from clinical treatment contacts is a much higher rate of injecting than would relate to amphetamine use in general, while the equivalent figure for cocaine was $24 \%$, indicating the prominence of the smokable crack form (Department of Health, 1995). The rise in crack cocaine was demonstrated by Strang et al (1990) who examined drug use in 441 attenders at a south London community drug team between 1987 and 1989, and found that in that period subjects using cocaine increased from $13 \%$ to $29 \%$, and within that group, use of smokable forms increased from $15 \%$ to $75 \%$.

Many drug misusers who use stimulants terminate episodes of use of such drugs, and alleviate withdrawal effects, by using various sedatives, including benzodiazepines, cannabis, or alcohol. This is reflected in DMD statistics and is commonly reported clinically, the most worrying combination being users of crack cocaine who turn to heroin for this purpose, with the result that they then become physically dependent on the opiate.

\section{Clinical features}

The main actions of amphetamine and cocaine resulting in a stimulant effect involve increased presynaptic release and inhibition of re-uptake of catecholamines, with a direct action on dopaminergic terminals and effects in the 'reward pathway' common to most drugs of misuse (Holman, 1994). The main clinical effects and withdrawal effects of stimulant drugs are shown in Box 1 . The effects are categorised into early effects, which are generally the desired effects, and the late features which most users recognise as indicating that they should terminate their episode of stimulant use.

Amphetamine has a slower onset of action than cocaine and a longer elimination half-life, and of the two forms of cocaine, crack has the quicker onset and withdrawal. The patterns of usage reflect this, and an amphetamine user may use the drug over a period of 2 to 3 days, going without sleep or much food. The undesirable effects then accumulate and he or she will stop the amphetamine, perhaps with the aid of sedatives as referred to above. By contrast, the whole process including height of action and experience of withdrawal effects is measured in a crack user in minutes rather than hours, and both aspects are usually much more intense.

The experience of paranoid feelings is very characteristic with both drugs, and is related to the complication of psychosis. As indicated, many users will stop at this stage, and in the relatively slow process of amphetamine symptoms it is debatable whether the subsequent depression, hyperphagia, and hypersomnia represent a true withdrawal syndrome or whether the last two features are simply catching up after a period without eating or sleeping. The similar more acute withdrawal effects from cocaine are often referred to as a 'crash', in which profound depression and craving are thought to be related to a rebound depletion in central dopamine transmission.

Gawin \& Kleber (1986) have described a threestage withdrawal syndrome in which these acute features are followed by more prolonged depressive symptoms, and then a normalisation of those features but the re-experience of withdrawal symptoms if exposed to 'cues' associated with previous usage. The entity of a withdrawal syndrome, however, still remains controversial, leading to these drugs generally being described as not physically addictive. The aspect of cues does appear important in relation to cocaine, in that individuals who have experienced severe with- 
drawal distress in their own situations may demonstrate few symptoms if admitted to hospital (Weddington et al, 1990).

\section{Complications}

One of the reasons that the massive recent US cocaine epidemic caused such concern was a high rate of medical complications, and stimulants also have the propensity to cause a range of psychiatric effects including psychosis. The main complications of amphetamine and cocaine misuse are indicated in Box 2.

The infective and general complications can occur in misuse of any drug which involves injecting or heavy usage. The cardiovascular complications are more specific to stimulant misuse, and clearly relate to the increase in catecholamine secretion. Stimulants cross the placenta, and although defining the links between drug misuse and obstetric complications is difficult research to do, as drug misusers tend to also be high risk in other ways such as diet, smoking, and poor living conditions, it appears that stimulants are more likely to be associated with such problems than most other drugs of misuse.

Irritability, agitation and depression have already been noted as withdrawal effects of stimulant drugs, and it is to some extent a matter of 'caseness' as to when such problems become clinical anxiety or depressive disorders. Similar problems of separation apply to antisocial behaviours such as aggressiveness, when there is the additional aspect that antisocial personality disorder is a frequent accompanying diagnosis in drug misusers (de Jong et al, 1993). It is difficult, but important, to distinguish in drug misusers between 'secondary' antisocial behaviours which may be related to the process of drug misuse itself, and definitely preexistent personality disorder, which requires a history predating drug misuse. The crack cocaine 'scene' is considered by enforcement authorities to be associated with particularly high levels of violence, which partly relates to aspects of dealing in this drug, where especially large amounts of money tend to be at stake, but which may also reflect desperate behaviour in those users who experience most acutely the intense withdrawal craving that can occur.

The best-known complication of stimulant misuse is a paranoid psychosis. Once again this can be seen as a development of the paranoid feelings that many stimulant users experience in their ordinary use of the drugs, but a full blown psychotic disorder seems to require heavy continuous usage. The paranoid delusions and associated features such as auditory hallucinations may exactly resemble schizophrenia, and indeed the fact that amphetamine can produce this was part of the evidence for the dopamine hypothesis of causation of schizophrenia. Tactile and visual hallucinations may occur and overactive, repetitive or compulsive behaviour may give a clue to stimulant use. Confusion and cognitive impairment are not usually present.

Urine screening for drugs of misuse should be done on young psychotic patients, and if stimulants are present another part of making the diagnosis of drug-induced psychosis is expectant management, as the psychosis may clear within a short number of days if the individual stops misusing drugs. Recently Poole \& Brabbins (1996) have criticised the diagnosis of drug-induced psychosis, observing that clear criteria are not usually applied, and that although relationships such as drugs worsening a functional psychosis, or individuals susceptible through family history developing psychosis after relatively small amounts, are likely, there is little proper evidence. It is important in differential diagnosis to remember that use of drugs which can cause psychosis such as amphetamines or cannabis is extremely widespread, and evidence of such use in an individual may not account for all of their symptoms, and also that other major drugs of misuse such as heroin do not have the propensity to cause psychosis at all.

\section{Management}

\section{Assessment}

The routine assessment of any form of drug misuse is indicated in Box 3. The points of history, examination and investigation mentioned relate to drug misuse itself rather than to its complications, for which additional assessment can be deduced. It is also important to establish a drug misuser's personal situation and the context in which they use drugs, and his or her motivation to change behaviour, including any external factors such as family pressure, ultimatum from partner, or legal trouble.

\section{Harm reduction}

Stimulant misuse is unfortunately not a form of drug misuse for which an obviously effective treatment exists, like methadone for heroin users. Also, not all users are inclined to completely stop using drugs, especially individuals who consider that their drug use has brought them no significant problems. Therefore it is especially necessary that measures are adopted aimed at reducing harm 
Box 2. Complications of amphetamine and cocaine misuse

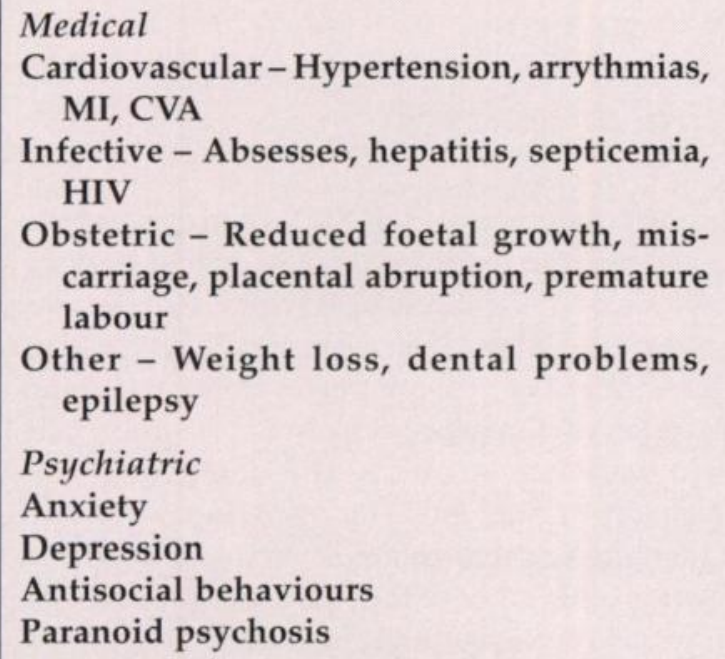

from drug misuse, including the general provision of information, education about health risks, advice to reduce damaging injecting practices, and the provision of clean injecting equipment. This work is usually done by community drug agencies, who will also provide counselling aimed at encouraging lifestyle and social changes. Members of drug misuse psychiatric teams, such as community psychiatric nurses or clinical psychologists, may counsel drug misusers in a more structured way, using behavioural methods to reduce usage, or the techniques of motivational interviewing.

\section{Pharmacological treatments}

There is a substantial literature from the US on medications which may reduce cocaine withdrawal symptoms, which has been reviewed by Withers et al (1995). A large number of compounds have been tried, which both reflects the scale of the US cocaine epidemic, but also the generally limited effectiveness of the medications involved. Because cocaine withdrawal effects are considered to be due to dopamine depletion, some trials have been of the dopaminergic agents, bromocryptine and amantadine, which have been found to reduce craving in some cases. Use of these drugs has not been significantly taken up in the UK, but there appears to be more use of desipramine, which is the most investigated drug in this indication in the US, where a meta-analysis of studies indicated significant benefit over placebo in promoting abstinence from cocaine (Levin \& Lehman, 1991). Carbamazepine is another medication which can reduce cocaine craving, and in general the same range of medications has been tried in the situation of reducing adjunctive cocaine use in patients on methadone. An alternative approach, again both in the acute withdrawal state and in attempting to achieve a general reduction in stimulant usage, is to use fluoxetine, as there is evidence, mainly from animal studies, that compounds which enhance serotonin transmission reduce consumption of substances of misuse, including stimulants. In the UK, fluoxetine has been found to help some amphetamine users reduce or stop their drug (Polson et al, 1993). Given the general difficulties in pharmacological management of drug misusers, these specialised treatments for which only limited evidence exists should probably only be used within drug misuse services.

In general, substitute prescribing for stimulant misusers is not considered suitable in the way that methadone is used for heroin users. This is because stimulants are inherently more destabilising drugs, and a true physical dependence is doubtful. While virtually nobody would support prescribing cocaine, some authorities in drug misuse feel that amphetamine prescribing should be considered for heavy daily users who appear unable to curtail their usage by any other method. Some services are experimenting with limited amphetamine prescribing, but the relatively short-acting dexamphetamine sulphate is not very satisfactory for the purpose.

\section{Treatments for psychiatric complications}

The management of psychiatric complications of stimulant misuse, including the use of antidepressants and antipsychotics, can be identical to treating the same disorders in other contexts. Medications are generally not effective in the face of ongoing drug misuse, and efforts must be directed to ensuring that this does not occur,

Box 3. Assessment of drug misuse

History

How often

How much

Routes of administration

Duration of usage

Significant drug-free periods

Previous treatment

Other associated drug misuse

Examination

Features of individual drugs

Injection marks

Investigation

Urine testing 
including frequent urine monitoring. Some specialists would consider that if an antidepressant is required, there is a theoretical advantage in using an SSRI, probably fluoxetine, while benzodiazepines should usually be avoided in managing anxiety symptoms.

\section{Admission}

Admission to a psychiatric ward, or sometimes alternatively to a drug rehabilitation centre, is indicated in three main situations. First, individuals in states of extreme withdrawal distress can require admission urgently, in a way that is hardly required in heroin users where methadone can effectively alleviate the situation. Secondly, there is a less urgent group of users who nevertheless feel unable to stop their drug use outside hospital in their own social situation, and request admission as the only way to successfully detoxify. It is important in assessing such cases to establish what is going to be different about the individual's social situation when they return to it, in order to attempt to avoid relapse. Finally, admission can be required for severe psychiatric complications, notably psychosis, especially if an individual lacks insight or is acting on delusional ideas.

\section{Other}

Because of the lack of definitely effective conventional treatments for stimulant misuse, such individuals may present for complementary therapies. The only systematic evidence for effectiveness relates to some limited benefits for acupuncture (Lipton et al, 1994).

\section{MDMA ('ecstasy')}

\section{Epidemiology}

MDMA was originally synthesised as an appetite suppressant, and was later used both as a recreational drug in US student circles, and as an aid to psychotherapy. Its current usage centres on 'rave' music parties in youth culture, notably in the UK. Recent surveys have found that $9 \%$ of 16-19 year olds and $8 \%$ of $16-29$ year olds had tried what they believed to be MDMA; analysis of drugs seized show the related chemicals MDA and MDEA to be gaining ground (Institute for the Study of Drug Dependence, 1994). It is estimated that there are approximately one million users of this group of drugs in the UK, and that most are weekend users, with a small minority progressing to more frequent use. The overlap with other drug usage occurs where amphetamine and LSD feature in the same youth culture, or where sedatives, rarely including heroin, are taken to terminate episodes of MDMA use.

\section{Clinical features}

The stimulant features of MDMA are as described above, but the drug also has so-called hallucinogenic, or psychedelic, effects which more resemble those of LSD. These include visual illusions, general enhancement of sensory perceptions, and states of altered consciousness, which are desired parts of the experience at raves. The most sought-after effect, which is achieved by many users of the drug, is a feeling of predominantly non-sexual affection towards others, hence the sobriquet 'the love drug'. Common physical effects include tachycardia, dry mouth, dilated pupils, facial muscle stiffness and parasthesiae.

Peroutka et al (1988) described effects of MDMA which occurred more than 24 hours after taking the drug, which become increasingly adverse and can broadly be seen as withdrawal effects. These can include tiredness, muscle aching, depression, headache and irritability.

\section{Complications}

Although large numbers of people use MDMA apparently unproblematically, there are specific complications which appear to relate to the complex pharmacology of the drug, which is well described by Steele et al (1994). Although complications in common with other stimulants may occur, particular interest has focused on deaths, and a range of psychiatric adverse effects.

\section{Deaths}

A number of deaths in recreational users have occurred (Henry et al, 1992; Milroy et al, 1996). It is notable that these do not seem particularly associated with heavy high dose usage, and neither is there reliable evidence of exposure to single doses only, as might be expected in a hypersensitivity reaction. Individuals have died with a range of pathology, including disseminated intravascular coagulation, rhabdomyolysis, renal and liver failure, shock, brain haemorrhages and pulmonary infarcts. A characteristic syndrome of hyperthermia is considered to relate both to the drug's direct effect on thermoregulation and also the environment in which MDMA is taken, involving vigorous non-stop dancing. One of the 
ways in which MDMA differs from other stimulants is that the effect on 5-HT neurones appears to be of more significance, and this transmitter is involved in internal heat regulation. It is not known why some individuals are susceptible to fatal effects, while even the common harm reduction advice to drink plenty of water when taking MDMA has proved controversial, given evidence in some cases of inappropriate anti-diuretic hormone secretion.

\section{Psychiatric disorders}

Although the systematic data largely relies on a series of case studies, it appears common for MDMA to cause psychiatric disturbance. A typical history is the individual who used MDMA recreationally over a period, possibly along with amphetamine or LSD, decided to stop, and "hasn't felt right since". In this context, clinicians are encountering anxiety states, depression, panic disorder, flashback experiences, and psychoses. The evidence for these is reviewed by Steele et al (1994) and by McGuire et al (1994), in which the link between MDMA and subsequent psychiatric problems in some of the cases is tenuous. Largerscale research needs to be done, but one feature that is emerging is that cases of psychiatric disorder which follow MDMA use are often resistant to treatment. MDMA is neurotoxic to 5-HT neurones, and so there is concern that resistance to treatment may reflect this form of brain damage.

\section{Management}

The general management of MDMA use resembles the approach to other forms of drug misuse. No specific treatments have been described, and for the psychiatric complications conventional medication should be used. Because one of the effects of MDMA is to cause a shut-down in 5-HT transmission, serotonergic antidepressants may be preferable, and may be useful when resistance to psychotropic treatments is encountered even if depressive features are not prominent.

\section{References}

De Jong, C. A. J., Van Den Brink, W., Harteveld, F. M., et al (1993) Personality disorders in alcoholics and drug addicts. Comprehensive Psychiatry, 34, 87-94.

Department of Health (1995) Statistical Bulletin: Drug Misuse Statistics 1995/24. London: HMSO.

Gawin, F. H. \& Kleber, H. D. (1986) Abstinence symptomatology and psychiatric diagnoses in cocaine abusers: clinical observations. Archives of General Psychiatry, 43, 107-113.
Henry, J. A., Jeffreys, K. J. \& Dawling, S. (1992) Toxicity and deaths from 3, 4-methylenedioxymethamphetamine ('ecstasy'). Lancet, 340, 384-387.

Holman, R. B. (1994) Biological effects of central nervous system stimulants. Addiction, 89, 1435-1441.

Institute for the Study of Drug Dependence (1994) Drug Misuse in Britain. London: ISDD.

Levin, F. R. \& Lehman, A. F. (1991) Meta-analysis of desipramine as an adjunct in the treatment of cocaine addiction. Journal of Clinical Psychopharmacology, 11, 374-378.

Lipton, D. S., Brewington, V. \& Smith, M. (1994) Acupuncture for crack-cocaine detoxification: experimental evaluation of efficacy. Journal of Substance Abuse Treatment, 11, 205-215.

McGuire, P. K., Cope, H. \& Fahy, T. A. (1994) Diversity of psychopathology associated with use of 3, 4-methylenedioxymethamphetamine ('Ecstasy'). British Journal of Psychiatry, 165, 391-395.

Milroy, C. M., Clark, J. C. \& Forrest, A. R. W. (1996) Pathology of deaths associated with "ecstasy" and "eve" misuse. Journal of Clinical Pathology, 49, 149-153.

Peroutka, S. J., Newman, H. \& Harris, H. (1988) Subjective effects of 3,4-methylenedixoymethamphetamine in recreational users. Neuropsychopharmacology, 1, 273-277.

Polson, R. G., Fleming, P. M. \& O'Shea, J. K. (1993) Fluoxetine in the treatment of amphetamine dependence. Human Psychopharmacology, 8, 55-58.

Poole, R. \& Brabbins, C. (1996) Drug induced psychosis. British Journal of Psychiatry, 168, 135-138.

Steele, T. D., McCann, T. D. \& Ricaurte, G. A. (1994) 3, 4methylenedioxymethamphetamine (MDMA, "ecstasy"): pharmacology and toxicology in animals and humans. Addiction, 89, 539-551.

Strang, J., Griffiths, P. \& Gossop, M. (1990) Crack and cocaine use in South London drug addicts: 1987-1989. British Journal of Addiction, 85, 193-196.

Weddington, W. W., Brown, B. S., Haertzen, C. A., et al (1990) Changes in mood, craving, and sleep during short-term abstinence reported by male cocaine addicts. Archives of General Psychiatry, 47, 861-868.

Withers, N. W., Pulvirenti, L., Koob, G. F., et al (1995) Cocaine abuse and dependence. Journal of Clinical Psychopharmacology, 15, 63-78.

\section{Multiple choice questions}

1 MDMA:

a has effects similar to those of amphetamine and LSD

b is a class A drug under the Misuse of Drugs Act

c is significantly misused by injection

d has adverse effects which can occur within 24 hours

e may cause depression

2 Withdrawal effects of stimulant drugs include:
a lacrimation
b agitation
c dilated pupils
d hypersomnia
e paranoid ideation

3 In a patient with paranoid psychosis whose urine shows amphetamine:

a amphetamine may be the cause of psychosis 
b amphetamine may be incidental to the psychosis

c antipsychotic medication must be avoided

d overactive behaviour suggests that the diagnosis is bipolar disorder

e daily urine monitoring should be performed

4 In the epidemiology of stimulant misuse:

a a high proportion of amphetamine users present to treatment services

b the Home Office Addicts Index only includes users of opiates, amphetamine and cocaine

c use of crack cocaine appears to be increasing

d surveys show that approximately $10 \%$ of under 29-year-olds have tried heroin

e it is rare for users of MDMA to use other drugs
5 'Crack' cocaine:

a is usually injected

b is made from cocaine hydrochloride

c can produce withdrawal features which include depression and craving

$\mathrm{d}$ is less pure than amphetamine powder

e may lead to violent behaviour

\section{MCQ answers}

\begin{tabular}{|c|c|c|c|c|c|}
\hline & 2 & 3 & & 4 & \\
\hline$T$ & a F & $\mathrm{a}$ & & & $\mathbf{F}$ \\
\hline$T$ & b $T$ & & $\mathbf{T}$ & b & F \\
\hline F & c $F$ & & $\mathbf{F}$ & & $\mathrm{T}$ \\
\hline 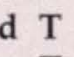 & d T & d & $\mathbf{F}$ & d & $F$ \\
\hline & e $F$ & e & $T$ & e & F \\
\hline
\end{tabular}

